\title{
Preparation of $\mathrm{SO}_{4}{ }^{2-} \mathrm{IZO}_{2}$ solid superacid and its efficient catalytic properties on the liquefaction of rapeseed straw
}

\author{
Xiaowei Han ${ }^{a}$, Runping $\mathrm{Jia}^{\mathrm{b}}$ \\ School of Materials Science and Engineering, Shanghai Institute of Technology, shanghai, 201418, \\ China \\ aemail: shuaisiyang@126.com, bemail:jiarp@sit.edu.cn
}

Keywords: Rapeseed Straw; Liquefaction; $\mathrm{SO}_{4}{ }^{2-} / \mathrm{ZrO}_{2}$ Solid Superacid; Biomass Polyols

\begin{abstract}
SO}_{4}{ }^{2-} / \mathrm{ZrO}_{2}$ solid superacid were prepared by the method of precipitation impregnation and the samples were characterized by SEM, XRD, and FT-IR. Then the samples were applied into the liquefied reaction of the waste rapeseed straw to prepare the biomass polyols and found it possessed efficient catalytic performance. And the catalytic liquefaction ratio of the waste rapeseed straw can reach $84 \%$. Moreover, the catalytic performance was investigated as a function of temperatures, reaction times, and catalyst contents, respectively.
\end{abstract}

\section{Introduction}

Biomass energy, like rapeseed straw, wheat straw, corn stover, bagasse, rice husk and so on, are very rich in China, but the utilization rate of biomass resources are low. Most of them have burn off direct without transformation into fuel with high calorific value, and caused serious environmental pollution. The shortage of energy and environmental pollution could be formed a serious threat to our country's economic security and sustainable development. Liquefaction of biomass is one of the effective methods for large-scale use of biomass. The liquefied products can be used as chemical raw material or fuel for comprehensive utilization. Thus it can reduce the consumption of fossil fuels instead of petroleum based products. The liquefaction of biomass has become an important way to prepare biobased materials [1].

The traditional liquefied technology of biomass for preparing the biobased polyols used polyols as liquefied reagent and concentrated sulfuric acid as catalyst to liquefy the waste biomass [2]. El-barbary M.Hasson et al. reported that they used PEG400 as the liquefied reagent to liquefy the bagasse and cotton stalks, and found that when they used PEG400 separately, the liquefaction ratio were low. Even under the best conditions, the liquefied residue rate of the bagasse and cotton stalks reached to $19 \%$ and $22 \%$, separately. When they added $10 \%$ glycerol, the liquefied residue rate reduced to $6.9 \%$ and $12.1 \%$, respectively [3] [4]. But using the concentrated sulfuric acid as catalyst to liquefy the waste biomass has many disadvantages, such as causing side effects in the process of liquefaction easily to recycle the raw materials difficultly, having strong corrosion resistance with higher requirements for the equipment, increasing the productive cost due to the complicated process, causing serious environmental pollution out of the emission of effluent and so on. Compared to the traditional concentrated sulfuric acid catalyst, the solid acid catalyst can overcome the above shortcomings [5]. The species of the solid acid are enormous, among them, the $\mathrm{SO}_{4}{ }^{2-} / \mathrm{M}_{\mathrm{x}} \mathrm{O}_{\mathrm{y}}$ solid superacids are preponderant on the aspects of preparation and preservation [6]. Especially, they have lots of advantages, such as higher catalytic activity, better selectivity, no corrosion to the equipment, no pollution to the environment and so on, compared to other solid acids. Thus, they are a kind of newly and potential catalytic materials [7]. Lifeng Yan et al. reported a novel process which they synthesized cellulose acetate in a solvent-free ball-milling reacter in the presence of solid superacid $\mathrm{SO}_{4}{ }^{2-} / \mathrm{ZrO}_{2}$ green catalyst and found that the maximum degree of substitution (DS) of formed cellulose acetate can achieve 1.8 [8].

Based on the description above, we had prepared the $\mathrm{SO}_{4}{ }^{2-} / \mathrm{ZrO}_{2}$ solid superacid by the method of precipitation impregnation as catalyst, and used waste rapeseed straw as raw materials and deionized water as liquefied reagent for catalytic liquefaction of the rapeseed straw in this study, 
and then used the liquefaction ratio of rapeseed straw as the index. We studied the influence of different factors on the liquefied effect of rapeseed straw by the method of single factor experiment systematically. We used the liquefied products as the chemical raw materials to prepare the polyurethane.

\section{Experimental}

$\mathrm{SO}_{4}{ }^{2-} / \mathrm{ZrO}_{2}$ solid superacid were prepared by the method of precipitation impregnation. $100 \mathrm{ml}$ $3 \mathrm{~mol} \cdot \mathrm{L}^{-1}$ solution of $\mathrm{Zr}\left(\mathrm{NO}_{3}\right)_{4}$ were prepared and pulled into the $250 \mathrm{ml}$ three flasks. Then the solution were mixed violently while dropping slowly $25 \%$ concentrated aqueous ammonia and adjusted $\mathrm{pH}$ to $9 \sim 10$. The mixture were continued to stir for $1 \mathrm{~h}$, and then ageing for $12 \mathrm{~h}$. The $\mathrm{Zr}(\mathrm{OH})_{4} \cdot \mathrm{nH}_{2} \mathrm{O}$ precipitation were acquired and washed repeatedly to no $\mathrm{NH}^{4+}$ with distilled water. Then the filter residue were dried at $110{ }^{\circ} \mathrm{C}$ and ground to 100 mesh. The dried products were the amorphous $\mathrm{ZrO}_{2}$ powder. According to the $15 \mathrm{ml} \cdot \mathrm{g}^{-1}$ ratio, they were immersed for $1 \mathrm{~h}$ with 0.5 mol $\cdot \mathrm{L}^{-1}$ sulfuric acid. After filtering and drying at $110{ }^{\circ} \mathrm{C}$, the filter residue were put into muffle furnace and dried at $550{ }^{\circ} \mathrm{C}$ for $3 \mathrm{~h}$. Then we acquired the $\mathrm{SO}_{4}{ }^{2-} / \mathrm{ZrO}_{2}$ solid superacid catalyst.

The biobased polyols were prepared by the method of the catalytic liquefaction of the waste rapeseed straw. According to the 1:10 scale, a certain amount rapeseed straw powder and deionized water were weighed and put into the open beaker. Then they were put into the microwave oven at $700 \mathrm{~W}$ for $1 \mathrm{~min}$. After the vacuum suction filter, they were dried in the oven at $90{ }^{\circ} \mathrm{C}$ for $6 \mathrm{~h}$. Then $1.5 \mathrm{~g}$ dried powder treated by the microwave oven, $50 \mathrm{~mL}$ deionized water and a small amount of homemade $\mathrm{SO}_{4}{ }^{2-} / \mathrm{ZrO}_{2}$ solid superacid catalyst were put into a $100 \mathrm{~mL}$ conical flask, and then mixed after ultrasound $5 \mathrm{~min}$. Then the mixture were put into the $100 \mathrm{~mL}$ hydrothermal reactor, and then put into the oven at a certain temperature for a period of time. After reaction and cooling down, the reaction mixtures were washed by ethanol and deionized water for many times. After the vacuum suction filter, The solid residues were put into the oven at $90{ }^{\circ} \mathrm{C}$ for $6 \mathrm{~h}$. After purified by vacuum distillation, the biomass polyols were extracted from the liquefied products.

\section{Result and Discussion}

Fig. 1 shows the SEM images of the $\mathrm{SO}_{4}{ }^{2-} / \mathrm{ZrO}_{2}$ solid superacid catalyst. We can clearly observe the samples were similar bar structure (Fig.1a). When we magnify the SEM image (Fig.1b), we can find the uniform granularity with length about $1 \mu \mathrm{m}$ and width about $200 \mathrm{~nm}$. Compared to the ordinary solid acid, they maybe possess a greater BET. These materials with particle size and appearance are beneficial to improve the reactive sites of catalytic liquefied reaction of the rapeseed straw and repeatable utilization.

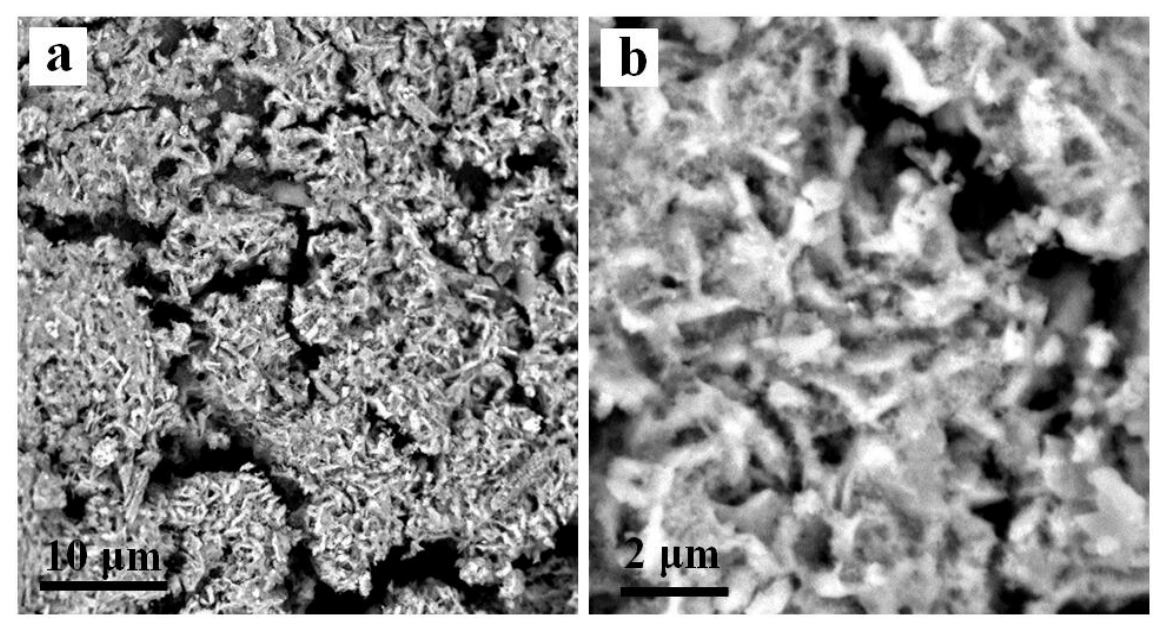

Fig.1. SEM images of the $\mathrm{SO}_{4}{ }^{2-} / \mathrm{ZrO}_{2}$ solid superacid catalyst 
Fig. 2 shows the XRD image of the $\mathrm{SO}_{4}{ }^{2-} / \mathrm{ZrO}_{2}$ solid superacid catalyst. The center strength of the $\mathrm{SO}_{4}{ }^{2-} / \mathrm{ZrO}_{2}$ solid superacid relate to its crystal shape and $\mathrm{ZrO}_{2}$ phase transformation. The sample as unique phase represented by reflections at $2 \theta=31.3^{\circ}, 33.5^{\circ}, 50.5^{\circ}, 60.2^{\circ}$. Then at $2 \theta=$ $28.4^{\circ}$, the decrease in the crystallite size of the monoclinic phase is due to the doping of zirconia with sulfate ions, which retard crystallite growth and keep the stabilization of zirconia tetragonal polymorph.

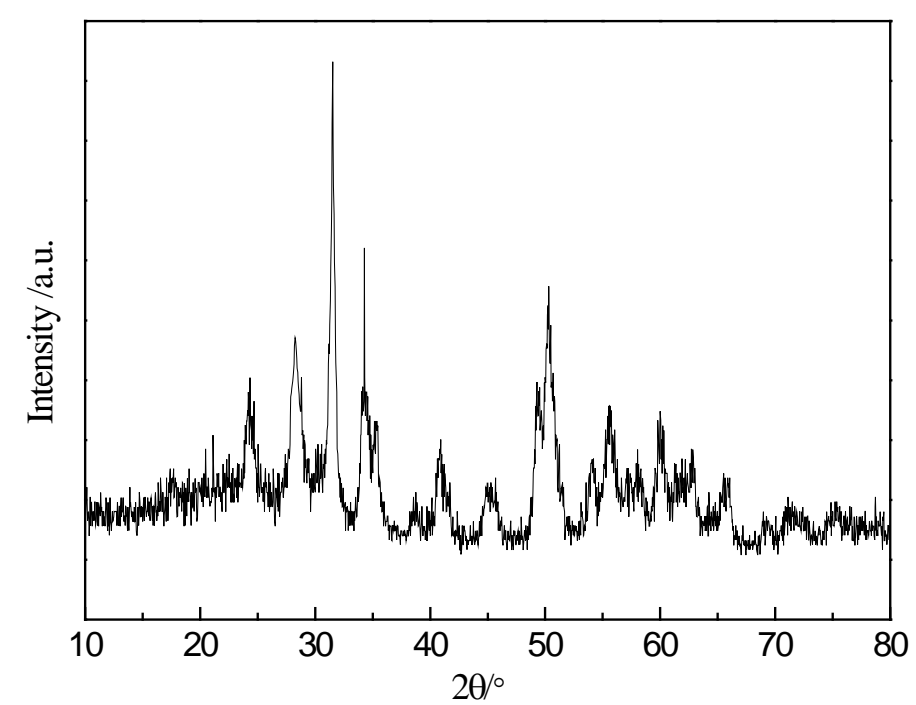

Fig.2. XRD image of the $\mathrm{SO}_{4}{ }^{2-} / \mathrm{ZrO}_{2}$ solid superacid catalyst

Fig.3 shows the FT-IR spectra of the $\mathrm{SO}_{4}{ }^{2-} / \mathrm{ZrO}_{2}$ solid superacid catalyst. We can obviously discover that the peak between $1110 \mathrm{~cm}^{-1}$ and $1162 \mathrm{~cm}^{-1}$ are the characteristic vibration of the M-O-S bond (Fig.2). The $\mathrm{S}=\mathrm{O}$ double bond's strong inductive effect results in the lack of electrons on the metal zirconium and reveals that a chemical bond between $\mathrm{SO}_{4}{ }^{2-}$ and $\mathrm{ZrO}_{2}$ has formed, just a Lewis(L acid) acid center. The very weak peak near $1400 \mathrm{~cm}^{-1}$ indicates the stretching vibration of the $\mathrm{S}=\mathrm{O}$ bond [9]. In conclusion, we have succeeded to prepare the $\mathrm{SO}_{4}{ }^{2-} / \mathrm{ZrO}_{2}$ solid superacid catalyst with high reaction and selection from the above images.

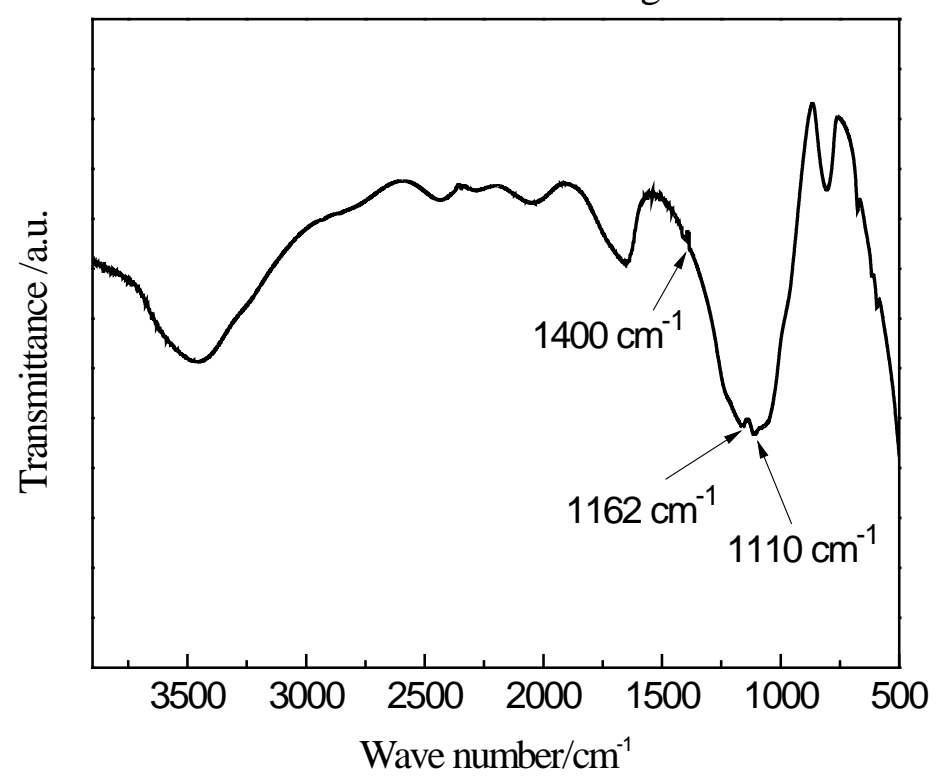

Fig.3. FT-IR spectra of the $\mathrm{SO}_{4}{ }^{2-} / \mathrm{ZrO}_{2}$ solid superacid catalyst

Fig. 4 shows the effect of different temperatures on the liquefaction ratio of the rapeseed straw. We can obviously catch that when the reaction temperature is lower than 200 degree (Fig.3), the catalytic effect of $\mathrm{SO}_{4}{ }^{2-} / \mathrm{ZrO}_{2}$ solid superacid catalyst are not obvious. With the increase of reaction temperature, the liquefaction ratio rise significantly and continuously. And when the reaction 
temperature reaches $240{ }^{\circ} \mathrm{C}$, the liquefaction ratio can get $84 \%$. Thus it can be seen, the reaction temperature has a great impact on the liquefaction of the rapeseed straw.

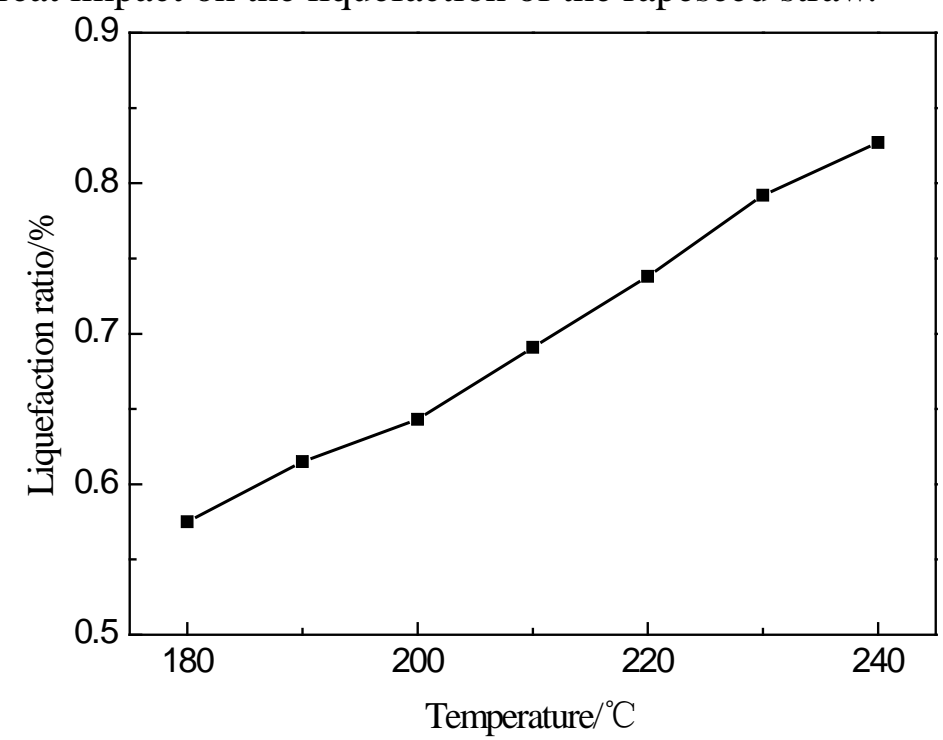

Fig.4. Effect of different temperatures on the liquefaction ratio of the rapeseed straw

Fig. 5 shows the effect of different reaction times on the liquefaction ratio of the rapeseed straw. We can obviously discovery that at the start of the reaction and before $2 \mathrm{~h}$, the liquefaction ratio increases significantly, then slowly (Fig.4). Then at the last part of liquefied reaction, the liquefaction ratio reduces instead. The main reason is that the small molecule compounds vaporize out of decomposition continuously, that result in the declination of the liquefaction ratio. So the best reaction time is $2 \mathrm{~h}$, and the liquefaction ratio can reach 64\%.

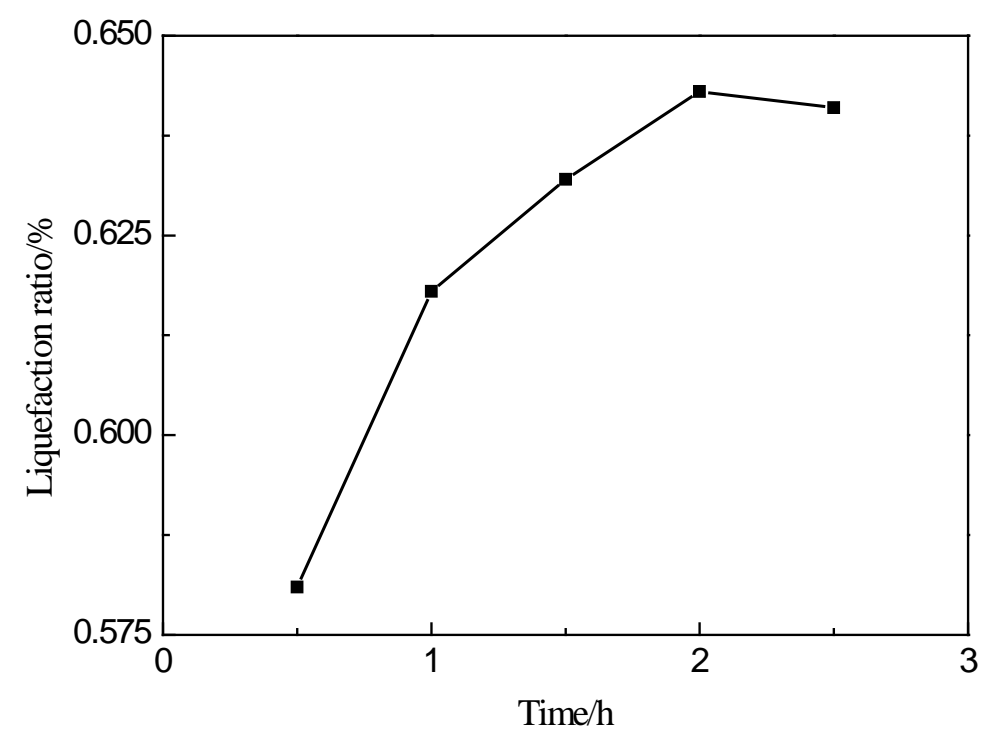

Fig.5. Effect of different reaction times on the liquefaction ratio of the rapeseed straw

Fig. 6 shows the effect of different catalyst contents on the liquefaction ratio of the rapeseed straw. We can obviously observe that before the catalyst contents reach $4 \%$ of the mass of the rapeseed straw, the liquefaction ratio increase continuously with the time (Fig.5). When the catalyst contents reach $4 \%$, the liquefaction ratio gets to the maximum $67 \%$. Then at the last part of liquefied reaction, the liquefaction ratio reduces instead. The main reason is that after the catalyst gets an excess, aggravating the decomposed production's condensate reaction results in increasing the residue and reducing the liquefaction ratio. 


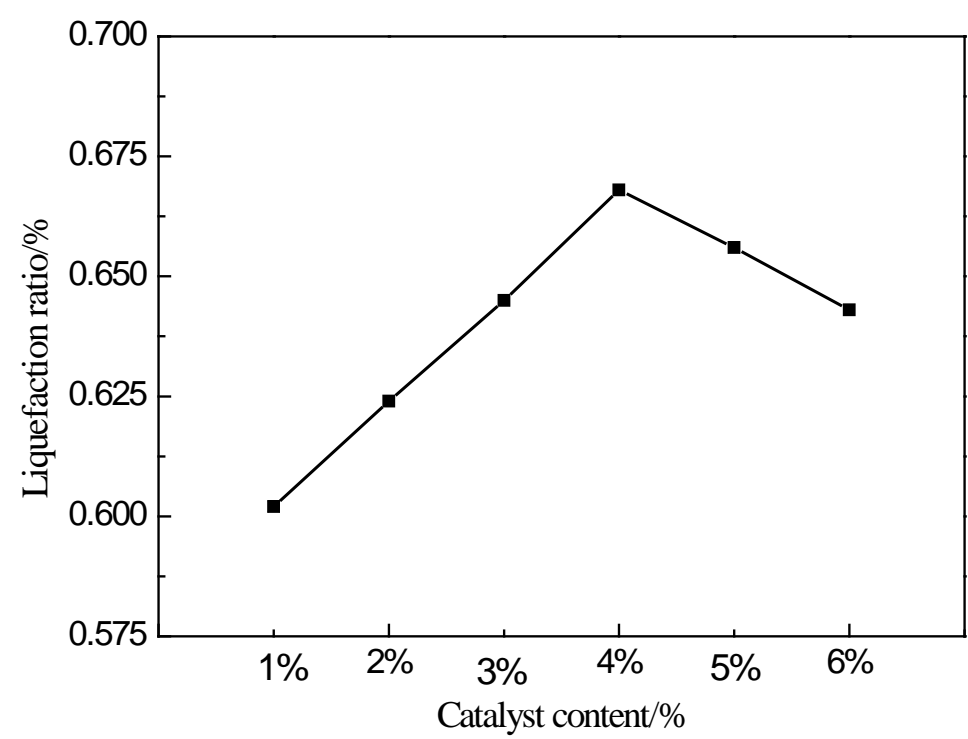

Fig.6. Effect of different catalyst contents on the liquefaction ratio of the rapeseed straw

\section{Conclusion}

In summary, we have successfully prepared $\mathrm{SO}_{4}{ }^{2-} / \mathrm{ZrO}_{2}$ solid superacid catalyst and applied to prepare the biobased polyols through the catalytic liquefaction of the waste rapeseed straw. In addition, we also found that the temperature is the most important factor of the catalytic liquefaction of the rapeseed straw. The results show that under the conditions which conclude $240{ }^{\circ} \mathrm{C}$ temperature, $2 \mathrm{~h}$ reaction time and $4 \%$ catalyst content, our liquefaction ratio can reach $84 \%$.

\section{Acknowledgement}

This work was financially supported by the Affiliate Programs. (LM201450).

\section{References}

[1] Tipeng Wang, Jun Yin, Zongming Zheng, Zhihuai Mao. Optimization of Reaction Conditions for Polyurethane Foam Synthesis with Liquefied Corn Stalk by Response Surface Methodology [J]. Journal of Applied Polymer Science, 2012, 125(125): 278-282.

[2] Jason D’Souza, Rafael Camargo, Ning Yan. Polyurethane Foams Made from Liquefied Bark-Based Polyols [J]. Journal of Applied Polymer Science, 2014, 131(16): 40599-40608.

[3] El-barbary M. Hasson, Nadia Shukry. Polyhydric alcohol liquefaction of some lignocellulosic agricultural residues [J]. Industrial Crops and Products, 2008, 27(1): 33-38.

[4] Rodrigo Briones, Luis Serrano, Jalel Labidi. Valorization of some lignocellulosic agro-industrial residues to obtain biopolyols [J]. Journal of Chemical Technology and Biotechnology, 2012, 87(2): 244-249.

[5] Qiang Zhang, Fangeng Chen, Lin Ma, Xuesong Zhou. Preparation and Application of Phosphorous-Containing Bio-Polyols in Polyurethane Foams [J]. Journal of Applied Polymer Science, 2014, 131(12): 40422-40427.

[6] Shaoyong Huang, Rufen Chen, Xuhong Zhang, Xiuqin Song. Preparation of Nanostructural $\mathrm{ZrO}_{2} / \mathrm{SiO}_{2}$ and Study on Catalytic Activity of its Superacid Catalyst [J]. Journal of the Chinese Chemical Society, 2007, 54(4): 997-1002. 
[7] Yong Wang, Shun Ma, Lili Wang, Shuze Tang, William W. Riley, Martin J. T. Reaney. Solid superacid catalyzed glycerol esterification of free fatty acids in waste cooking oil for biodiesel production [J]. European Journal of Lipid Science and Technology, 2012, 114(3): 315-324.

[8] Lifeng Yan, Wan Li, Zhonghui Qi, Shaoyang Liu. Solvent-free Synthesis of Cellulose Acetate by Solid Superacid Catalysis [J]. Journal of Polymer Research, 2006, 13(5): 375-378. 